\section{Effect of \\ trabeculectomy \\ on RNFL thickness \\ and optic disc \\ parameters using \\ optical coherence tomography}

N Raghu, SS Pandav, S Kaushik, P Ichhpujani

and A Gupta postoperatively following glaucoma filtration surgery. RNFL thickness temporarily increased and cup area decreased but the values reverted to normal within 3 months. Eye (2012) 26, 1131-1137; doi:10.1038/eye.2012.115; published online 22 June 2012

Keywords: optical coherence tomography; trabeculectomy; RNFL thickness; optic disc

\section{Introduction}

Reversal of optic disc cupping following intraocular pressure (IOP) reduction is a wellknown phenomenon in congenital ${ }^{1,2}$ and juvenile ${ }^{3,4}$ glaucoma. Although it has been reported in adult patients also, the results of these studies seem conflicting. The stage of disease, the amount of IOP reduction, and the age of the patient probably influence the occurrence of this event, resulting in inconsistent outcomes. The clinical relevance of 'reversal' has not been established with certainty, although reports ${ }^{5,6}$ have suggested that there may be an associated improvement of visual function that corresponds to this improvement in disc appearance.

Clinical examination of the optic disc is often poorly reproducible ${ }^{7,8}$ and subtle changes in the optic nerve head $(\mathrm{ONH})$ topography and retinal nerve fibre layer (RNFL) may be difficult to assess. The main site of glaucoma damage is believed to be at the lamina cribrosa, and the mechanical theory of glaucoma damage postulates that raised IOP can cause a backward bowing of the lamina, resulting in damage of the nerve fibres passing thorough this
Advanced Eye Centre, Postgraduate Institute of Medical Education and Research, Chandigarh, India

Correspondence: SS Pandav, Advanced Eye Centre, Postgraduate Institute of Medical Education and Research, Sector 12, Chandigarh 160012, India Tel: +91 172 2747837; Fax: +91 1722747837 . E-mail: sspandav@yahoo.com

Received: 27 October 2011 Accepted in revised form: 13 February 2012 Published online: 22 June 2012 
structure. ${ }^{9,10}$ Reduction of the IOP should theoretically ease this back bowing, and maybe the mechanism of reversal of optic disc cupping even in adults. Release of pressure on the nerve fibres passing through the lamina may also result in changes in RNFL thickness after IOP reduction.

The Heidelberg retinal tomogram (HRT) has been used to assess the ONH following IOP reduction in adults but the results are not uniform. Some studies have shown reversal of optic disc cupping that was proportional to the magnitude of IOP reduction ${ }^{11-14}$ and age. ${ }^{15}$ However, there are other studies that have shown this reversal was a transient phenomenon, which reverted to baseline values with time. ${ }^{16}$ Others have shown no significant change in optic disc topography. ${ }^{17}$

The RNFL thickness after IOP reduction has been studied using optical coherence tomography (OCT) ${ }^{18-20}$ and GDx. ${ }^{21,22}$ These reports are conflicting as well. Early studies using the $\mathrm{OCT}^{18}$ and $\mathrm{GDx}^{21}$ showed increased RNFL thickness following trabeculectomy. However, later studies ${ }^{19,20,22}$ showed no change in RNFL thickness on OCT following IOP reduction.

The OCT can also measure optic disc topography. Although there are inherent limitations in the measurement algorithm, the discriminating ability of some parameters for glaucoma has been shown to be nearly as good as the RNFL thickness measurements. ${ }^{23,24}$ OCT may be more advantageous over HRT in having a higher axial resolution and automated outlining of optic disc margin, thus eliminating operator variablity. ${ }^{25}$ One added advantage of the OCT ONH analysis is that, one can get a cross-sectional view of the ONH rather than the 'enface' view provided by the HRT. If cup reversal following IOP reduction is due to the forward movement of the lamina cribrosa, then OCT may be able to demonstrate this phenomenon better.

Our study looked at both change in RNFL thickness measurements and ONH topography by OCT in order to demonstrate any structural improvement following IOP reduction in adult patients.

\section{Materials and methods}

This was a prospective interventional study including consecutive adult patients ( $>18$ years) with uncontrolled glaucoma requiring surgical treatment presenting to the Glaucoma Clinic of the Advanced Eye Centre, Postgraduate Institute of Medical Education and Research, Chandigarh, India. Subjects fulfilling the inclusion criteria detailed below were prospectively recruited for the study. The study obtained approval from the Institute Ethics Committee, and adhered to the principles of the Declaration of Helsinki. Informed consent was obtained from all the recruited individuals.
Each subject underwent a comprehensive ophthalmic examination including best-corrected visual acuity (BCVA) recorded as LOGMAR values, IOP measured by Goldmann Applanation tonometry, slit lamp biomicroscopy, gonioscopy, and stereoscopic fundus evaluation on the slit lamp using a 90.0-D lens. Colour stereoscopic optic disc photographs and red-free nerve fibre layer photographs were taken on the Zeiss Fundus camera FF 450 with Visupac System 451 (Carl Zeiss Ophthalmic Systems, GmBH, Jena, Germany). Subjects with visual acuity permitting the test underwent standard achromatic perimetry on the Humphrey's Field Analyser HFA 750 II, (Carl Zeiss Humphrey Systems, Dublin, CA, USA) using the 24-2 testing protocol by SITA-Standard strategy. The visual fields were considered satisfactory if false positive and false negative errors did not exceed $30 \%$ and fixation errors did not exceed $25 \%$.

Any form of glaucoma was eligible for inclusion, including primary and secondary glaucoma. Patients $>18$ years of age, with unsatisfactory IOP control for the status of their glaucoma while on maximum tolerable anti-glaucoma medications, who were scheduled for glaucoma-filtering surgery, were enrolled for the study.

Patients were excluded if they had any history of ocular disease or intraocular surgery, or if any ocular disease was detected during examination, such as diabetic retinopathy, uveitis, or cataract. Any eye with media haze precluding view of second-order retinal vessels or any patient unable to undergo the procedure under local anaesthesia was also excluded from the study. If both eyes of a subject were eligible for the study, the eye with the clearer media was enrolled to ensure the best possible OCT images.

All eligible subjects underwent baseline and postoperative RNFL thickness and optic disc measurement using the Zeiss Optical Coherence Tomographer Stratus OCT version 4.0.1 (Carl Zeiss Meditec Ophthalmic Systems Inc., Dublin, CA, USA). All OCT scans were acquired by an experienced operator (SK). OCT scans were obtained after pupillary dilatation using $5 \%$ phenylephrine and $1 \%$ tropicamide drops instilled once or twice as required. All baseline scans were to be acquired within 2 weeks of the planned surgery.

\section{OCT scanning}

The machine was properly aligned after seating the subject with the chin comfortably resting on the chin rest. The OCT lens was adjusted for the patient's refractive error. The contralateral eye was covered. All scans were done using the internal fixation target in the OCT device. The subject was instructed to fixate with the eye being 
tested on the internal target, to enable the $\mathrm{ONH}$ to come into focus. The Z-Offset was adjusted to view the OCT image, and polarization was optimized to maximize the reflective signal. An average of three readings was computed in each case. To be acceptable for inclusion, the OCT scans had to fulfil the following criteria: the fundus image must be clear enough to see the optic disc and scan circle or spokes, the scans must be properly centred on the optic disc, the signal strength should be $>5$ (prescribed as the minimum-acceptable criteria in the Stratus OCT manufacturer operation manual), ${ }^{14}$ colour saturation must be even and dense across the entire scan, and there must be red colour visible in the RPE and RNFL with proper detection of RNFL boundaries by the software. Care was taken to ensure no missing areas in the scan due to blinks or eye motion.

- The peripapillary RNFL was scanned using the fast RNFL thickness (3.4) scanning protocol, which uses an aiming circle with a default diameter measuring $3.46 \mathrm{~mm}$. The operator manually adjusted this aiming circle such that its centre coincided with the centre of the optic disc, ensuring an OCT image of even radius of $1.73 \mathrm{~mm}$ around the $\mathrm{ONH}$.

- The optic disc was imaged using the fast optical disc scanning protocol. This protocol acquires six $4.0 \mathrm{~mm}$ equally spaced line scans spaced at $30^{\circ}$ to each other running through a common central axis, placed in the centre of the optic disc. The Stratus OCT automatically detects the ends of the $\mathrm{ONH}$ as the end of the retinal pigment epithelium/choriocapillaris, with a provision of manual correction of this assessment if not satisfactory. All optic disc scans were checked for the accuracy of the automated detection of the optic disc margins. Any inaccuracies were manually corrected by the operator and the scans were analysed using the corrected determination.

The OCT images were analysed with the in-built Stratus OCT software (Carl Zeiss Meditec Ophthalmic Systems Inc.). The RNFL parameters studied were average RNFL thickness and RNFL thickness in each of four quadrants defined as superior $\left(46-135^{\circ}\right)$, nasal $\left(136-225^{\circ}\right)$, inferior $\left(226-315^{\circ}\right)$, and temporal $\left(316-45^{\circ}\right)$. The ONH parameters studied were rim area, cup area, horizontal integrated rim width (HIRW), and cup-disc area ratio.

\section{Surgical technique}

All glaucoma-filtering surgeries were trabeculectomy with limbus-based conjunctival flaps and adjuvant injection mitomycin $C$, and were performed by the same surgeon (SSP) using similar surgical technique in all the patients. A limbal-based conjunctival flap was dissected. Tenon's capsule was separated by dissecting between the capsule and episclera. The sclera was exposed and haemostasis was achieved by wet-field cautery. A partial thickness triangular scleral flap ( $4 \mathrm{~mm}$ base and $4 \mathrm{~mm}$ height) was prepared using lamellar dissection.

Mitomycin C $(0.4 \%)$ was used as an adjuvant for $2 \mathrm{~min}$ under the scleral flap before entering anterior chamber. Residual MMC was washed with copious amount of fluid. Sclerostomy $(3 \times 1.5 \mathrm{~mm})$ was done by removing a block of trabecular tissue at the sclerolimbal junction. Peripheral iridectomy was performed through the sclerostomy. The scleral flap was sutured with one fixed suture at the apex and two releasable 10-0 nylon sutures at the sides of the flap. Conjunctiva and tenons were closed in separate layers using 10-0 vicryl continuous sutures.

Any intraoperative or postoperative complications associated with the procedure were noted.

Postoperative visual acuity, IOP, slit lamp biomicroscopy, and disc evaluation were done at day 1 , day 2 , week 1 , week 2 , fortnightly for a month, and then as required thereafter. OCT scans and disc photography was repeated at 1 week, 1 month, and 3 months postoperatively.

All measurements were computed on prospectively filled data forms.

\section{Statistical analysis}

All analyses were performed with SPSS software (version 12; SPSS Inc., Chicago, IL, USA) and $P$-values $<0.05$ were considered to be statistically significant. The Wilcoxon signed-rank test was used to analyse the differences in IOP and variation in RNFL thickness and $\mathrm{ONH}$ parameters before and after glaucoma surgery. Spearman's correlation was used to correlate percentage change in IOP with the percentage change observed in the OCT parameters. The mean deviation on visual fields was also correlated to the percentage change in RNFL and $\mathrm{ONH}$ parameters to determine whether the stage of glaucoma was a factor in any structural change following IOP reduction.

\section{Results}

A total of 22 eyes of 22 patients met the inclusion criteria. Three patients were excluded due to less than optimal OCT scans. Two patients did not return for the 3-month follow-up visit. In all, 17 eyes of 17 Asian Indian patients were analysed. There were 13 male and 4 female patients; mean age was $51.23 \pm 7.15$ years. Seven patients had primary open angle glaucoma, six patients had primary angle closure glaucoma, three patients had angle 
recession glaucoma, and one patient had juvenile open angle glaucoma. Most patients had advanced glaucoma at presentation. The average SD on visual fields was $-20.4 \pm 8.6 \mathrm{~dB}$.

Preoperative scans were acquired within 1 week (6.93 \pm 1.27 days) before surgery; the postoperative 1 -week scan was acquired at $6.73 \pm 1.27$ days, 1 -month scan at $31.06 \pm 3.01$ days, and 3-month scan was acquired at $90 \pm 7.20$ days from the date of surgery.

Pre- and postoperative visual acuity and IOP are shown in Figure 1, and OCT parameters are shown

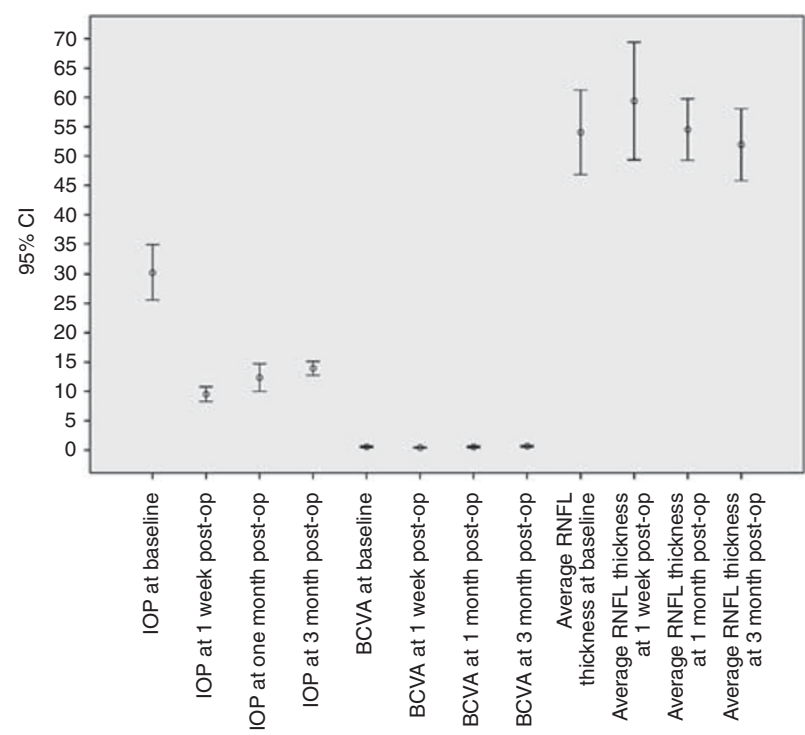

Figure 1 Error bar diagram showing mean IOP, BCVA, and average retinal nerve fibre layer (RNFL) thickness measurements at baseline, 1 week, 1 month, and 3 months post trabeculectomy. The error bars represent the $95 \%$ confidence limits. in Table 1 . The BCVA decreased transiently at 1 week post surgery, but there was no difference in pre- and postoperative visual acuity after that. The mean preoperative IOP was $30.2 \pm 9.0 \mathrm{~mm} \mathrm{Hg}$, which decreased significantly $(P<0.001)$ to $9.5 \pm 2.4 \mathrm{~mm} \mathrm{Hg}$, $12.3 \pm 4.6 \mathrm{~mm} \mathrm{Hg}$, and $13.9 \pm 2.3 \mathrm{~mm} \mathrm{Hg}$ at 1 week, 1 month, and 3 months postoperatively, respectively.

RNFL and optic disc measurements on the OCT are depicted in Table 1. The mean preoperative average RNFL thickness was $54.05 \pm 14.02 \mu \mathrm{m}$, which increased significantly to $59.39 \pm 19.52 \mu \mathrm{m}(P=0.009)$ at 1 week postoperatively, but decreased to $54.50 \pm 10.17$ and $51.95 \pm 11.94 \mu \mathrm{m}$ at 1 and 3 months postoperatively. Among the quadrant measurements, the inferior and temporal RNFL thickness increased by $6.76 \pm 12.34 \mu \mathrm{m}$ $(P=0.023)$ and $8.47 \pm 17.3 \mu \mathrm{m}(P=0.044)$, respectively, at the first postoperative week, but decreased to baseline values in the subsequent visits. There was no significant change from baseline values in the other quadrant measurements.

Among the $\mathrm{ONH}$ parameters, the cup area decreased significantly at 1 week from $2.33 \pm 0.5 \mathrm{~mm}^{2}$ preoperatively to $2.0 \pm 0.61 \mathrm{~mm}^{2}$ at 1 week $(P=0.014)$, but reverted close to baseline values by 3 months (Table 1). The cup-disc area ratios decreased significantly from preoperative $0.87 \pm 0.13$ to $0.73 \pm 0.19$ at 1 week $(P=0.005)$, but the change was not significant at 1 month $(0.79 \pm 0.15 ; P=0.246)$ and 3 months $(0.81 \pm 0.16 ; P=0.309)$ follow-up. There was no significant change in the rim area or HIRW measurements postoperatively.

The IOP reduction was $66.8 \%$ at 1 week, $55.9 \%$ at 1 month, and $49.9 \%$ at 3 months postoperatively. The corresponding percentage change in average RNFL thickness was $13.4 \%, 7.6 \%$, and $9.5 \%$ at 1 week, 1 month,

Table 1 Change in RNFL and ONH parameters on the Stratus OCT following trabeculectomy

\begin{tabular}{|c|c|c|c|c|c|c|c|}
\hline \multirow[t]{2}{*}{ OCT Parameters } & \multirow[t]{2}{*}{ Preoperative (mean $\pm S D)$} & \multicolumn{6}{|c|}{ Postoperative (mean $\pm S D)$} \\
\hline & & 1 Week & $\mathrm{P}^{\mathrm{a}}$ & 1 Month & $\mathrm{P}^{\mathrm{a}}$ & 3 Months & $\mathrm{P}^{\mathrm{a}}$ \\
\hline \multicolumn{8}{|l|}{ RNFL thickness $(\mu \mathrm{m})$} \\
\hline Average & $54.1 \pm 14.0$ & $59.4 \pm 19.5$ & 0.009 & $54.5 \pm 10.2$ & 0.193 & $51.9 \pm 11.9$ & 0.619 \\
\hline Superior & $59.9 \pm 17.7$ & $64.9 \pm 24.5$ & 0.136 & $60.1 \pm 15.2$ & 0.679 & $59.5 \pm 17.7$ & 0.717 \\
\hline Inferior & $60.3 \pm 20.6$ & $67.1 \pm 22.2$ & 0.023 & $61.1 \pm 16.6$ & 0.426 & $56.3 \pm 14.2$ & 0.185 \\
\hline Nasal & $57.4 \pm 17.7$ & $58.3 \pm 20.7$ & 0.652 & $55.1 \pm 15.7$ & 0.448 & $52.4 \pm 19.5$ & 0.149 \\
\hline Temporal & $38.6 \pm 11.3$ & $47.1 \pm 23.5$ & 0.044 & $42.6 \pm 14.4$ & 0.477 & $39.3 \pm 10.1$ & 0.619 \\
\hline \multicolumn{8}{|l|}{ ONH parameters } \\
\hline Rim area $\left(\mathrm{mm}^{2}\right)$ & $0.44 \pm 0.29$ & $0.55 \pm 0.5$ & 0.758 & $0.51 \pm 0.44$ & 0.981 & $0.48 \pm 0.32$ & 0.795 \\
\hline Cup area $\left(\mathrm{mm}^{2}\right)$ & $2.33 \pm 0.5$ & $2.00 \pm 0.61$ & 0.014 & $2.19 \pm 0.58$ & 0.084 & $2.25 \pm 0.59$ & 0.554 \\
\hline HIRW (mm) & $0.79 \pm 0.25$ & $0.79 \pm 0.38$ & 0.962 & $0.65 \pm 0.27$ & 0.356 & $0.76 \pm 0.26$ & 0.653 \\
\hline Cup-disc area ratio & $0.87 \pm 0.13$ & $0.73 \pm 0.19$ & 0.005 & $0.79 \pm .0 .15$ & 0.246 & $0.81 \pm 0.16$ & 0.309 \\
\hline
\end{tabular}

Abbreviations: HIRW: horizontal integrated rim width; ONH: optic nerve head; RNFL: retinal nerve fibre layer.

a The Wilcoxon signed-rank test. 
and 3 months, respectively. The percentage change in mean cup-disc area ratio was $18.8 \%, 11.7 \%$, and $14.8 \%$ at 1 week, 1 month, and 3 months, respectively. There was no correlation between percentage change in IOP and change in RNFL and optic disc parameters. We also correlated percentage change in these parameters with age but found no significant correlation. There was no relationship observed between the $\mathrm{MD}$ at baseline and percentage change in RNFL thickness or ONH parameters.

\section{Discussion}

It is believed that the main cause of glaucomatous damage is compression of neurons by the distorted, posteriorly bowed lamina cribrosa, resulting in loss of nourishment to retinal ganglion cells. ${ }^{9,10}$ This distortion may be permanent in advanced cases, but there is mounting evidence that in less advanced disease, both the disc and visual field can improve when the IOP is lowered. ${ }^{26,27}$ It is postulated that one of the main reasons for an apparent improvement in optic nerve appearance with IOP reduction is a reduction in the posterior bowing of the lamina cribrosa, giving relief to these compressed nerve fibre bundles. ${ }^{10}$ Apparent improvement in the appearance of the $\mathrm{ONH}$ has been well documented in children. ${ }^{1-4}$ However, it has still been a relatively infrequently described phenomenon in adults. One of the reasons maybe that techniques like $\mathrm{ONH}$ photography may not be sensitive enough to detect the small changes occurring. Unless the photographs are carefully examined, subtle alterations are likely to be missed, and the subjective nature of the examination is also a limiting factor. The ability to examine optic discs by image analysis techniques has improved the ability to recognize changes in the $\mathrm{ONH}$ more objectively, and with greater accuracy.

We used the OCT for topographical assessment of the optic disc. ONH analysis by the Stratus OCT has been shown to have high reproducibility, with the manually corrected fast scan having the lowest coefficient of variation. ${ }^{28}$ We used the manually corrected fast scan for our topographical analysis also. The cross-sectional scan imaged by the OCT gave a better representation of the anterior movement of the lamina cribrosa than could have been appreciated by the enface image acquired by the HRT. The structural reversal of optic disc parameters following reduction in IOP has not been studied using the OCT previously.

There are studies investigating changes in optic disc topography demonstrated by the HRT, ${ }^{11-17}$ and changes in the RNFL thickness by OCT ${ }^{18-20}$ and GDx ${ }^{21,22}$ following reduction in IOP, albeit with conflicting conclusions. The variable results of these studies may be due to other factors such as stage of the disease and age.

Our study showed that the cup area and cup-disc area ratio decreased transiently 1 week after surgery but reverted to close to baseline values by 3 months. Among the RNFL parameters, average and inferior and temporal quadrant RNFL thickness measurements increased significantly at 1 week after surgery, but reverted to baseline levels at the subsequent follow-up visits.

Several studies have reported reduction in cupping after IOP reduction following glaucoma surgery. ${ }^{11-17}$ This observation is likely owing to a simple shift in anatomic structures rather than recovery or reversal of damage. When the IOP is lowered, there is less stretch on the lamina cribrosa of the disc that probably causes the return of the laminar surface to its previous position before the raised IOP caused it to bow back. This return may manifest as decreased cup area postoperatively.

In contrast to our study, Irak et al ${ }^{11}$ reported significant changes in several optic disc parameters on the HRT 4.5 months after surgery, which had a significant association with the IOP reduction. Likewise, Kotecha et al ${ }^{13}$ reported persistent reversal of disc cupping following trabeculectomy 2 years following IOP reduction. Our study agrees with the findings of Topouzis et $a l^{16}$ who found no difference in most optic disc parameters 4 months after surgery, and the cup shape also reverted to baseline values 8 months after surgery. The IOP reduction in our study $(49.9 \pm 16.8 \%)$ is similar to Irak et $a l^{11}$ and Topouzis et $a l^{16}(43.8 \% \pm 29.9 \%$ and $49.3 \pm 14.9 \%$, respectively), whereas Emery et al ${ }^{9}$ had IOP reduction of $36.62 \pm 18.64 \%$. However, most of our patients had advanced glaucoma (MD - 20.4 $\pm 8.6 \mathrm{~dB}$ ), which might have accounted for more irreversible changes at the level of the ONH. The patients in Topouzis' study had average preoperative MD $-13.2 \pm 6.8 \mathrm{~dB}$, while Kotecha's group had moderate glaucoma (MD $-9.9 \pm 8.4 \mathrm{~dB}$ ). This may have been a factor in the conflicting results, as experimental studies have shown that the earlier the stage of glaucoma, the more likely it is that reversal of cupping may occur. ${ }^{29,30}$ The transient early postoperative changes observed in our study might have well been the effects of postoperative hypotony.

Reported RNFL thickness change following IOP reduction has also shown conflicting results. Chang et $a l^{19}$ used scanning laser polarimetry and found a significant increase in RNFL thickness 3-6 months following trabeculectomy, but this had no correlation with IOP. However, this study was performed using the older generation GDx, which has the potential for greater inter-individual and intra-individual variability as it lacks the correction for corneal polarization. Using the 
newer GDx machine, Koraszewska-Matuszewska and Samochowiec-Donocik ${ }^{22}$ reported no change in RNFL thickness in juvenile glaucoma patients following trabeculectomy.

Aydin $e a^{18}$ used a noncommercial, prototype OCT device to evaluate RNFL thickness change following trabeculectomy, and reported a significant increase. It has been shown that the sensitivity and specificity for RNFL thickness measurement using the prototype device is less as compared with the newer version. ${ }^{31}$ One possible explanation could be that they included patients undergoing cataract surgery also. Although they did analyse RNFL changes in the trabeculectomy alone group, the differences were more marked in the group that had the cataract surgery also, and there was no difference in the superior and inferior quadrant thickness in the trabeculectomy group. Improvement in signal strength itself may yield thicker measurements of RNFL, and this may have had a role in the thicker RNFL measured postoperatively in patients undergoing combined surgery.

Subsequent studies using the Stratus OCT for RNFL thickness measurement as in this study, show similar results as ours. Chang et $a l^{19}$ showed no significant change in the RNFL thickness associated with the lowering of IOP by medical or surgical therapy. Rebolleda et $a^{20}$ reported no change in peripapillary RNFL thickness 6 months following deep sclerectomy. They separately analysed patients with $<30 \%$ and $>50 \%$ IOP reduction, and reported no change in either group.

A possible explanation suggested for the immediate increase in RNFL thickness postoperatively is the reversal or rebound of the physical compressive effect on the RNFL by the elevated pre intervention IOP, leading to a recovery of normal shape and size by the retinal ganglion cell axons. ${ }^{10}$ Another explanation that has been postulated may be retinal swelling from acute postoperative reduction in IOP. ${ }^{9}$

The current study shows that the RNFL thickness increases and cup area decreases for a transient period after glaucoma surgery but reverts back to preoperative values within 3 months. No correlation was seen between IOP change and RNFL thickness as well as cup area. At least in patients with advanced glaucoma, disc and RNFL changes appear to be irreversible, and lowering IOP substantially does not appear to result in any structural improvement in the $\mathrm{ONH}$ or peripapillary RNFL parameters. There appears to be no need to redefine baseline values, and preoperatively established values can be used for follow-up after 3 months of surgery. Further studies on patients presenting with earlier stage of the disease might answer the question of whether structural glaucoma damage is reversible if intervened in time.

\section{Summary}

What was known before

- RNFL thickness was thought to increase after reduction of IOP. Later reports showed there was no difference after some time. There was no information of $\mathrm{ONH}$ parameters measured by the OCT following IOP reduction. There was no clear information of these changes in predominantly advanced glaucoma.

\section{What this study adds}

- This study was mainly done in advanced glaucoma patients. It has shown prospectively an initial increase in RNFL thickness following trabeculectomy, followed by reversal of these changes to baseline values after 3 months. It adds information regarding the change in optic nerve parameters documented on the OCT, showing reversal of all apparent improvement by 3 months.

\section{Conflict of interest}

The authors declare no conflict of interest.

\section{References}

1 Meirelles SH, Mathias CR, Bloise RR, Stohler NS, Liporaci $\mathrm{SD}$, Frota AC et al. Evaluation of the factors associated with the reversal of the disc cupping after surgical treatment of childhood glaucoma. J Glaucoma 2008; 17(6): 470-473.

2 Yasuda M, Ando A, Otsuji T, Fukui C, Matsumura M. Improvement of the topographic parameters of the optic discs after trabeculotomy in two patients with developmental glaucoma. Eye 2006; 20(1): 132-134.

3 Leung CK, Woo J, Tsang MK, Tse KK. Structural and functional recovery in juvenile open angle glaucoma after trabeculectomy. J Pediatr Ophthalmol Strabismus 2009; 46(6): 372-375.

4 Wu SC, Huang SC, Kuo CL, Lin KK, Lin SM. Reversal of optic disc cupping after trabeculotomy in primary congenital glaucoma. Can J Ophthalmol 2002; 37(6): 337-341.

5 Tsai CS, Shin DH, Wan JY, Zeiter JH. Visual field global indices in patients with reversal of glaucomatous cupping after intraocular pressure reduction. Ophthalmology 1991; 98(9): 1412-1419.

6 Leung CK, Woo J, Tsang MK, Tse KK. Structural and functional recovery in juvenile open angle glaucoma after trabeculectomy. Eye 2006; 20(1): 132-134.

7 Parrish RK II, Schiffman JC, Feuer WJ, Anderson DR, Budenz DL, Wells-Albornoz MC et al. Ocular Hypertension Treatment Study Group. Test-retest reproducibility of optic disk deterioration detected from stereophotographs by masked graders. Am J Ophthalmol 2005; 140(4): 762-764.

8 Azuara-Blanco A, Katz LJ, Spaeth GL, Vernon SA, Spencer $\mathrm{F}$, Lanzl IM. Clinical agreement among glaucoma experts in the detection of glaucomatous changes of the optic disk using simultaneous stereoscopic photographs. Am J Ophthalmol 2003; 136(5): 949-950.

9 Emery JM, Landis D, Paton D, Boniuk M, Craig JM. The lamina cribrosa in normal and glaucomatous human eyes. Trans Am Acad Ophthalmol Otolaryngol 1974; 78: 290-297. 
10 Quigley HA, Addicks EM, Green WR, Maumenee AE. Optic nerve damage in human glaucoma. II. The site of injury and susceptibility to damage. Arch Ophthalmol 1981; 99: 635-649.

11 Irak I, Zangwill L. Garden V, Shakiba S, Weinreb RN. Change in optic disk topography after trabeculectomy. Am J Ophthalmol 1996; 122(5): 690-695.

12 Raitta C, Tomita G, Vesti E, Harju M, Nakao H. Optic disc topography before and after trabeculectomy in advanced glaucoma. Ophthalmic Surg Lasers 1996; 27(5): 349-354.

13 Kotecha A, Siriwardena D, Fitzke FW, Hitchings RA, Khaw PT. Optic disc changes following trabeculectomy: longitudinal and localisation of change. $\mathrm{Br} J$ Ophthalmol 2001; 85(8): 956-961.

14 Park KH, Kim DM, Youn DH. Short-term change of optic nerve head topography after trabeculectomy in adult glaucoma patients as measured by Heidelberg retina tomograph. Korean J Ophthalmol 1997; 11(1): 1-6.

15 Lesk MR, Spaeth GL, Azuara-Blanco A, Araujo SV, Katz LJ, Terebuh A. Reversal of optic disc cupping after glaucoma surgery analyzed with a scanning laser tomography. Ophthalmology 1999; 106: 1013-1018.

16 Topouzis F, Peng F, Kotas-Neumann R, Garcia R, Sanguinet $\mathrm{J}$, Yu F et al. Longitudinal changes in optic disc topography of adult patients aftertrabeculectomy. Ophthalmology 1999; 106(6): 1147-1151.

17 Paranhos A Jr, Lima MC, Salim S, Caprioli J, Shields MB. Trabeculectomy and optic nerve head topography. Braz J Med Biol Res 2006; 39(1): 149-155.

18 Aydin A, Wollstein G, Price LL, Fujimoto JG, Schuman JS. Optical coherence tomography assessment of retinal nerve fiber layer thickness changes after glaucoma surgery. Ophthalmology 2003; 110(8): 1506-1511.

19 Chang PT, Sekhon N, Budenz DL, Feuer WJ, Park PW, Anderson DR. Effect of lowering intraocular pressure on optical coherence tomography measurement of peripapillary retinal nerve fiber layer thickness. Ophthalmology 2007; 114(12): 2252-2258.

20 Rebolleda G, Muñoz-Negrete F, Noval S. Evaluation of changes in peripapillary nerve fiber layer thickness after deep sclerectomy with optical coherence tomography. Ophthalmology 2007; 114: 488-493.

21 Yamada N, Tomita G, Yamamoto T, Kitazawa Y. Changes in the nerve fiber layer thickness following a reduction of intraocular pressure after trabeculectomy. I Glaucoma 2000; 9(5): 371-375.

22 Koraszewska-Matuszewska B, Samochowiec-Donocik E. Evaluation of retinal nerve fiber layer thickness in eyes with juvenile glaucoma after trabeculectomy. Klin Oczna 2004; 106: 443-444.

23 Medeiros FA, Zangwill LM, Bowd C, Vessani RM, Susanna $\mathrm{R} \mathrm{Jr}$, Weinreb RN. Evaluation of retinal nerve fiber layer, optic nerve head, and macular thickness measurements for glaucoma detection using optical coherence tomography. Am J Ophthalmol 2005; 139: 44-55.

24 Wollstein G, Ishikawa H, Wang J, Beaton SA, Schuman JS. Comparison of three optical coherence tomography scanning areas for detection of glaucomatous damage. Am J Ophthalmol 2005; 139: 39-43.

25 Leung CK, Chan WM, Hui YL, Yung WH, Woo J, Tsang MK et al. Analysis of retinal nerve fiber layer and optic nerve head in glaucoma with different reference plane offsets, using optical coherence tomography. Invest Ophthalmol Vis Sci 2005; 46(3): 891-899.

26 Tsai CS, Shin DN, Wan JY, Zeiter JH. Visual filed global indices in patients with reversal of glaucomatous cupping after intraocular pressure reduction. Ophthalmology 1991; 98: 1412-1419.

27 Katz LJ, Spaeth GL, Cantor LB, Poryzees EM, Steinmann WC. Reversible optic disc cupping and visual field improvement in adults with glaucoma. Am J Ophthalmol 1989; 107: 485-492.

28 Kammppeter BA, Kristin V, Budde WM, Degenring RF, Jonas JB. OCT of optic nerve head interindividual reproducibility. J of glaucoma 2006; 15: 248-254.

29 Shirakashi M, Nanba K, Iwata K. Changes in reversal of cupping in experimental glaucoma. Ophthalmology 1992; 99: 1104-1110.

30 Coleman AL, Quigley HA, Vitale S, Dunkelberger G. Displacement of the optic nerve head by acute changes in intraocalr pressure in monkey eyes. Ophthalmology 1991; 98: 35-40.

31 Bourne RR, Medeiros FA, Bowd C, Jahanbakhsh K, Zangwill LM, Weinreb RN. Comparability of retinal nerve fibre layer thickness measurements of optical coherence tomography instruments. Invest Ophthalmol Vis Sci 2005; 46: 1280-1285. 